\title{
Clinical features and prognosis-associated factors of non-small cell lung cancer exhibiting symptoms of bone metastasis at the time of diagnosis
}

\author{
YI-FU HE ${ }^{1,2^{*}}$, HUI-QIN LUO ${ }^{*}$, WEI WANG ${ }^{1}$, JIAN CHEN $^{1}$, YI-WEI YAO $^{1}$, SHAN-BAO CAI ${ }^{3}$, JIE HE $^{4}$, YING YAN ${ }^{2}$, \\ SHU-SHENG WU ${ }^{2}$, XIAO-XIU HU ${ }^{2}$, LI-HONG KE ${ }^{2}$, JIA-YU NIU ${ }^{2}$, HUI-MIN LI ${ }^{2}, \mathrm{CHU}_{-S H U} \mathrm{JI}^{1}$ and BING HU ${ }^{1}$ \\ ${ }^{1}$ Department of Medical Oncology, Anhui Provincial Hospital Affiliated to Anhui Medical Univeristy; \\ Departments of ${ }^{2}$ Medical Oncology, ${ }^{3}$ Orthopedics and ${ }^{4}$ Pathology, Anhui Provincial Cancer Hospital, \\ Hefei, Anhui 230001, P.R. China
}

Received June 6, 2014; Accepted February 17, 2015

DOI: $10.3892 / \mathrm{ol} .2015 .3081$

\begin{abstract}
The present study aimed to analyze the clinical characteristics and prognosis-related factors of non-small cell lung cancer (NSCLC) patients with bone metastases at the time of diagnosis. A total of 46 NSCLC patients with skeletal metastases at the time of diagnosis from Anhui Provincial Hospital and Anhui Provincial Cancer Hospital Affiliated to Anhui Medical University (Hefei, China) between February 2010 and February 2012 were investigated retrospectively. The median age was 58 years, with a range of 40-80 years, the ratio of males and females was $2: 1$, and adenocarcinoma and squamous cell carcinoma accounted for 71.7 and $28.3 \%$ of cases, respectively. Furthermore, $84.8 \%$ of patients exhibited multiple skeletal metastases at more than two sites and $54.3 \%$ of patients experienced skeletal-related events at the time of diagnosis. The median overall survival (OS) time of the patients was 237 days, and Kaplan-Meier analysis demonstrated that patients with adenocarcinoma $(\mathrm{P}=0.002)$, single bone metastases $(\mathrm{P}=0.023)$, an Eastern Cooperative Oncology Group performance status of $0-1 \quad(\mathrm{P}<0.001)$ or positive expression of estrogen receptor $(\mathrm{ER})-\beta(\mathrm{P}=0.039)$ exhibited significantly longer survival times. Furthermore, multivariate analysis identified the following independent predictors of OS: Tumor subtype $(\mathrm{P}=0.022)$, the number of bone metastases $(\mathrm{P}=0.016)$ and an ER- $\beta$-positive tumor $(\mathrm{P}=0.035)$. In the cohort of NSCLC patients with bone metastases at the time of diagnosis, adenocarcinoma and multiple skeletal metastases were most common.
\end{abstract}

Correspondence to: Professor Bing Hu, Department of Medical Oncology, Anhui Provincial Hospital Affiliated to Anhui Medical University, 17 Lujiang Road, Hefei, Anhui 230001, P.R. China

E-mail: hubing3756@hotmail.com

${ }^{*}$ Contributed equally

Key words: bone metastases, non-small cell lung cancer, clinical characteristics, prognostic factors, estrogen receptor- $\beta$

\section{Introduction}

Lung cancer is currently the most common and most fatal type of malignant tumor in China (1). The incidence rate of bone metastasis from lung cancer is as high as 30-40\% (2). In particular, skeletal metastases acutely affect the quality of life, with one study revealing the median survival time of lung cancer with bone metastases to be only 6-10 months (3). In recent years, extended survival times have been observed in patients with lung cancer following the application of targeted therapeutic agents; however, longer survival is associated with a higher incidence of bone metastases. Non-small cell lung cancer (NSCLC) patients with bone metastases at the time of diagnosis require study, as few reports regarding the clinical characteristics and prognosis of such patients are available. Beattie et al (4) identified lung cancer as a hormone-dependent tumor as early as 1985. It was subsequently determined that the level of estrogen receptor (ER)- $\beta$ expression in lung cancer was significantly higher compared with that in healthy individuals, and that the expression levels were closely associated with prognosis (5). However, to the best of our knowledge, no reports have thus far been conducted with regard to the expression of ER- $\beta$ in NSCLC patients with bone metastases at the time of diagnosis. Therefore, the aim of the present study was to analyze the ER- $\beta$ expression levels, as well as the clinical and survival characteristics, of 46 cases of NSCLC with bone metastases at the time of diagnosis, and to attempt to associate the current findings with prognostic evaluation and clinical treatment.

\section{Patients and methods}

Patients. The present cohort included 57 NSCLC patients exhibiting skeletal metastases at the time of diagnosis, including 41 cases from Anhui Provincial Hospital Affiliated to Anhui Medical University (Hefei, China) and 16 cases from Anhui Provincial Cancer Hospital Affiliated to Anhui Medical University, between February 2010 and February 2012. Among the 57 patients, incomplete data was obtained from 6 patients and 5 patients refused treatment. These patients were excluded, therefore, 46 patients were included in the present analysis. 

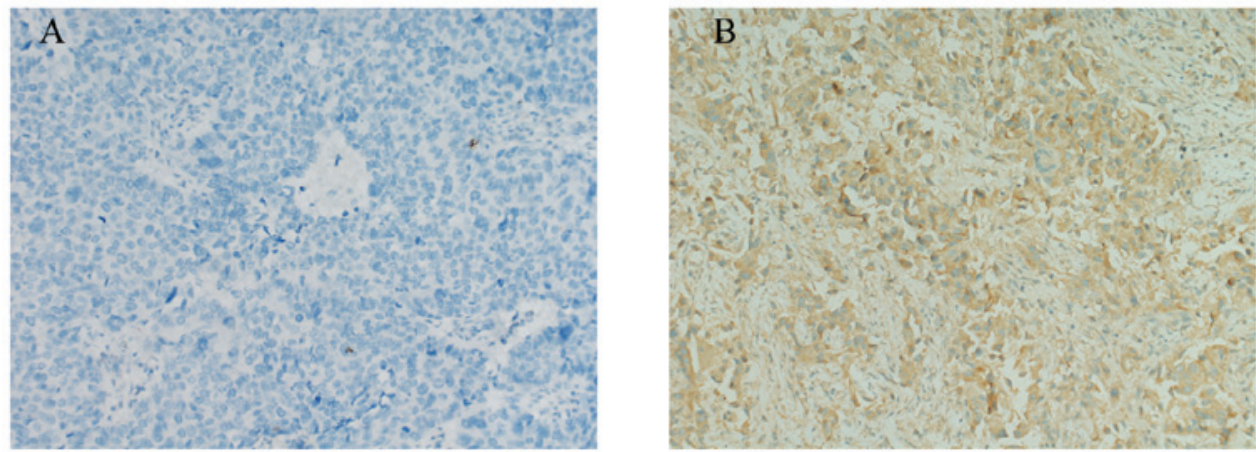

Figure 1. Immunohistochemical detection of estrogen receptor (ER)- $\beta$ expression in non-small cell lung cancer patients with bone metastases at the time of diagnosis (labeled streptavidin-biotin staining; magnification, $x 200)$. (A) ER- $\beta$ negative expression. (B) ER- $\beta$ positive expression as brown cytoplasmic staining.

This study was approved by the ethics committee of Anhui Provincial Hospital Affiliated to Anhui Medical University and written informed consent was obtained from all of the patients.

Definition of bone metastases and diagnosis of skeletal-related events (SRES). Bone metastases in NSCLC were defined using the following criteria: i) Pathological diagnosis $(n=14)$, pathological examination was performed with tissues from the metastatic sites; ii) imaging diagnosis $(n=46)$, emission computed tomography (ECT) indicated multiple bone metastases; ECT was positive and confirmed by CT, magnetic resonance imaging or X-ray; ECT was positive and accompanied with clinical symptoms, such as local pain or tenderness, pathological fracture or paraplegia; or positron emission tomography-CT indicated bone metastases; and iii) concurrent pathological and imaging diagnosis $(n=14)$. SREs were defined as a series of events caused by bone metastases of the malignant tumor, including severe bone pain, pathological fracture, spinal cord compression and hypercalcemia.

\section{Therapeutic regimens}

Treatment of lung cancer. The predominant treatment strategy was chemotherapy combined with best supportive care due to the advanced stage of the patients upon diagnosis. The first-line chemotherapy regimens administrated were platinum plus gemcitabine/pemetrexed/vinorelbine. Regimen 1: Gemcitabine, $1000 \mathrm{mg} / \mathrm{m}^{2}$ ivgtt d1d8 combined with cisplatin, $75 \mathrm{mg} / \mathrm{m}^{2}$ (7 patients) or carboplatin, $5 \mathrm{mg} / \mathrm{m}^{2}$ ivgtt d2 (5 patients). Regimen 2: Paclitaxel, $150 \mathrm{mg} / \mathrm{m}^{2}$ ivgtt d1 in combination with cisplatin, $75 \mathrm{mg} / \mathrm{m}^{2}$ ivgtt $\mathrm{d} 2$, (17 cases). Regimen 3: Navelbine, $25 \mathrm{mg} / \mathrm{m}^{2}$ iv d1d5 combined with cisplatin, $75 \mathrm{mg} / \mathrm{m}^{2}$ ivgtt d1 (17 cases). All patients agreed to receive a minimum of two cycles of chemotherapy. A total of 151 cycles of chemotherapy was used, average of 2-6 cycles.

Treatment of bone metastases. A minimum of one round of bisphosphonate therapy (one dose per month of zoledronic acid, $4 \mathrm{mg} / \mathrm{month}$ ). was administered for all patients and analgesics were applied for patients with cancer-associated pain. In addition, local radiotherapy (dose for one patient, $50 \mathrm{~Gy}$ ) was performed to control pain in 9 patients and surgical fixation was performed in 5 patients.

Immunohistochemistry. Paraffin-embedded tissue sections (thickness, $4 \mu \mathrm{m}$ ) of NSCLC were deparaffinized, heated in an oven for $2 \mathrm{~h}$ at $60^{\circ} \mathrm{C}$ and immunohistochemically stained using immunohistochemistry autostainer (Benchmark XT; Roche Pharmaceutical Ltd., Basel, Switzerland). ER- $\beta$ kits were purchased from Beijing Bioss Biosynthesis Biotechnology Co., Ltd., (Beijing, China) and positive ER- $\beta$ staining was indicated by the presence of tan-colored particles in the nucleus or cytoplasm. Positive and negative control tests were used in each experiment; PBS was used as a negative control and ovarian tissue used as the positive control of ER- $\beta$ expression.

Additional clinical parameters. Age, gender, pathological type, smoking status, the number of bone metastases, SREs, whether other organs metastases existed, Eastern Cooperative Oncology Group (ECOG) performance status score (6), and serum calcium, carcinoembryonic antigen (CEA) and alkaline phosphatase (ALK) levels were recorded as additional clinical parameters.

Statistical analysis. Survival rates were calculated using the Kaplan-Meier method. Survival time was defined as from the date of diagnosis until the date of mortality, and the median follow-up period was 24 months. The log-rank method was used for univariate analysis and the Cox regression model was used for multivariate analysis, with differences between the groups analyzed by $\chi^{2}$ test. $\mathrm{P}<0.05$ was considered to indicate a statistically significant difference and all analyses were performed using SPSS software for Windows (version 16.0; SPSS, Inc., Chicago, IL, USA).

\section{Results}

Patient characteristics. The median age of the 46 patients investigated was 58 years (range, $40-80$ years) and the gender ratio was 2:1 (male, 31 cases; female, 15 cases). The baseline characteristics of all patients are shown in Table I.

Immunohistochemical results. ER- $\beta$-positive expression was identified in the cytoplasm of the cells obtained from the NSCLC patients with bone metastases at the time of diagnosis, as indicated in Fig. 1 . The total ER- $\beta$-positive rate was 78.3 and $74.2 \%$ in the male and female patients; 78.9 and $77.8 \%$ in the patients aged $\geq 60$ and $<60$ years old; 81.8 and $69.2 \%$ in the adenocarcinoma and squamous cell carcinoma 
Table I. Clinical characteristics and univariate analysis of 46 non-small cell lung cancer patients with bone metastases at the time of diagnosis.

\begin{tabular}{|c|c|c|c|c|}
\hline \multirow[b]{2}{*}{ Characteristic } & \multirow[b]{2}{*}{ Patients, n (\%) } & \multicolumn{3}{|c|}{ Overall survival time, days } \\
\hline & & Median & $95 \% \mathrm{CI}$ & P-value \\
\hline \multicolumn{5}{|l|}{ Age, years } \\
\hline$\geq 60$ & $19(41.3)$ & 226 & $184-268$ & 0.665 \\
\hline$<60$ & $27(58.7)$ & 269 & $218-320$ & \\
\hline \multicolumn{5}{|l|}{ Gender } \\
\hline Male & $31(67.4)$ & 227 & $152-302$ & 0.101 \\
\hline Female & $15(32.6)$ & 238 & $185-291$ & \\
\hline \multicolumn{5}{|l|}{ Smoker } \\
\hline Yes & $23(50.0)$ & 209 & $159-287$ & 0.133 \\
\hline No & $23(50.0)$ & 273 & $160-257$ & \\
\hline \multicolumn{5}{|l|}{ Histology } \\
\hline Adenocarcinoma & $33(71.7)$ & 273 & $192-354$ & 0.002 \\
\hline Squamous carcinoma & $13(28.3)$ & 158 & $45-270$ & \\
\hline \multicolumn{5}{|l|}{ Skeletal metastases, $\mathrm{n}$} \\
\hline 1 & $7(15.2)$ & 428 & $372-485$ & 0.023 \\
\hline$\geq 2$ & $39(84.8)$ & 227 & $193-261$ & \\
\hline \multicolumn{5}{|l|}{ SREs } \\
\hline Yes & $25(54.3)$ & 273 & $188-358$ & 0.830 \\
\hline No & $21(45.7)$ & 198 & $148-248$ & \\
\hline \multicolumn{5}{|c|}{ Other existing organ metastases } \\
\hline Yes & $29(63.0)$ & 231 & $193-269$ & 0.322 \\
\hline No & $17(37.0)$ & 273 & $101-445$ & \\
\hline \multicolumn{5}{|l|}{ ECOG score } \\
\hline $0-1$ & $20(43.5)$ & 330 & $257-403$ & 0.001 \\
\hline $2-4$ & $26(56.5)$ & 158 & $71-245$ & \\
\hline \multicolumn{5}{|l|}{ ALK, U/1 } \\
\hline$>150$ & $12(26.1)$ & 231 & $217-244$ & 0.668 \\
\hline$\leq 150$ & $34(73.9)$ & 237 & $160-314$ & \\
\hline \multicolumn{5}{|l|}{ CEA, ng/l } \\
\hline$\leq 5$ & $34(73.9)$ & 238 & $181-295$ & 0.290 \\
\hline$\leq 5$ & $12(26.1)$ & 215 & $81-349$ & \\
\hline \multicolumn{5}{|l|}{$\mathrm{ER}-\beta$} \\
\hline Positive & $36(78.3)$ & 269 & $215-323$ & 0.039 \\
\hline Negative & $10(21.7)$ & 196 & $108-284$ & \\
\hline
\end{tabular}

SREs, skeletal-related events; ECOG, Eastern Cooperative Oncology Group; ALK, alkaline phosphatase; CEA, carcinoembryonic antigen; ER- $\beta$, estrogen receptor- $\beta$; CI, confidence interval.

cases; 85.7 and $76.9 \%$ in the single and multiple bone metastases groups; 79.3 and $76.4 \%$ in the patients with other organ metastases and no other metastases; and 73.5 and $91.7 \%$ in the normal and elevated ALK groups, respectively. Furthermore, no statistically difference in ER- $\beta$-positive expression was identified between each group using the $\chi^{2}$ test.

Univariate analysis. The median survival time of the patients in the present study was 237 days. Univariate analysis identified that pathological type $(\mathrm{P}=0.002)$, the number of bone metastases $(\mathrm{P}=0.023)$ and the ECOG score $(\mathrm{P}<0.001)$ at the time of diagnosis, and the ER- $\beta$ expression status $(\mathrm{P}=0.039)$ were prognostic factors of NSCLC with bone metastases at the time of diagnosis. Specifically, patients with squamous cell carcinoma, with greater than two sites of bone metastases, an ECOG performance status of 2-4 and an ER- $\beta$-negative tumor exhibited a poor prognosis. By contrast, gender, smoking status, position of bone metastases, SREs, other organ metastases, and serum ALK and CEA levels were not significantly correlated with the prognosis of the patients (Table I; Fig. 2). 
Table II. Multivariate analysis of non-small cell lung cancer patients with bone metastases at the time of diagnosis.

\begin{tabular}{lccr}
\hline & \multicolumn{2}{c}{ Overall survival } & P-value \\
\cline { 2 - 3 } Independent prognostic factor & Odds ratio & $95 \%$ CI & 0.022 \\
\hline Pathological type & 3.449 & $1.196-9.943$ & 0.016 \\
Number of skeletal metastases & 4.146 & $1.303-13.186$ & 0.035 \\
ER- $\beta$ expression status & 0.396 & $0.167-0.939$ & \\
\hline
\end{tabular}

CI, confidence interval; ER- $\beta$, estrogen receptor- $\beta$.
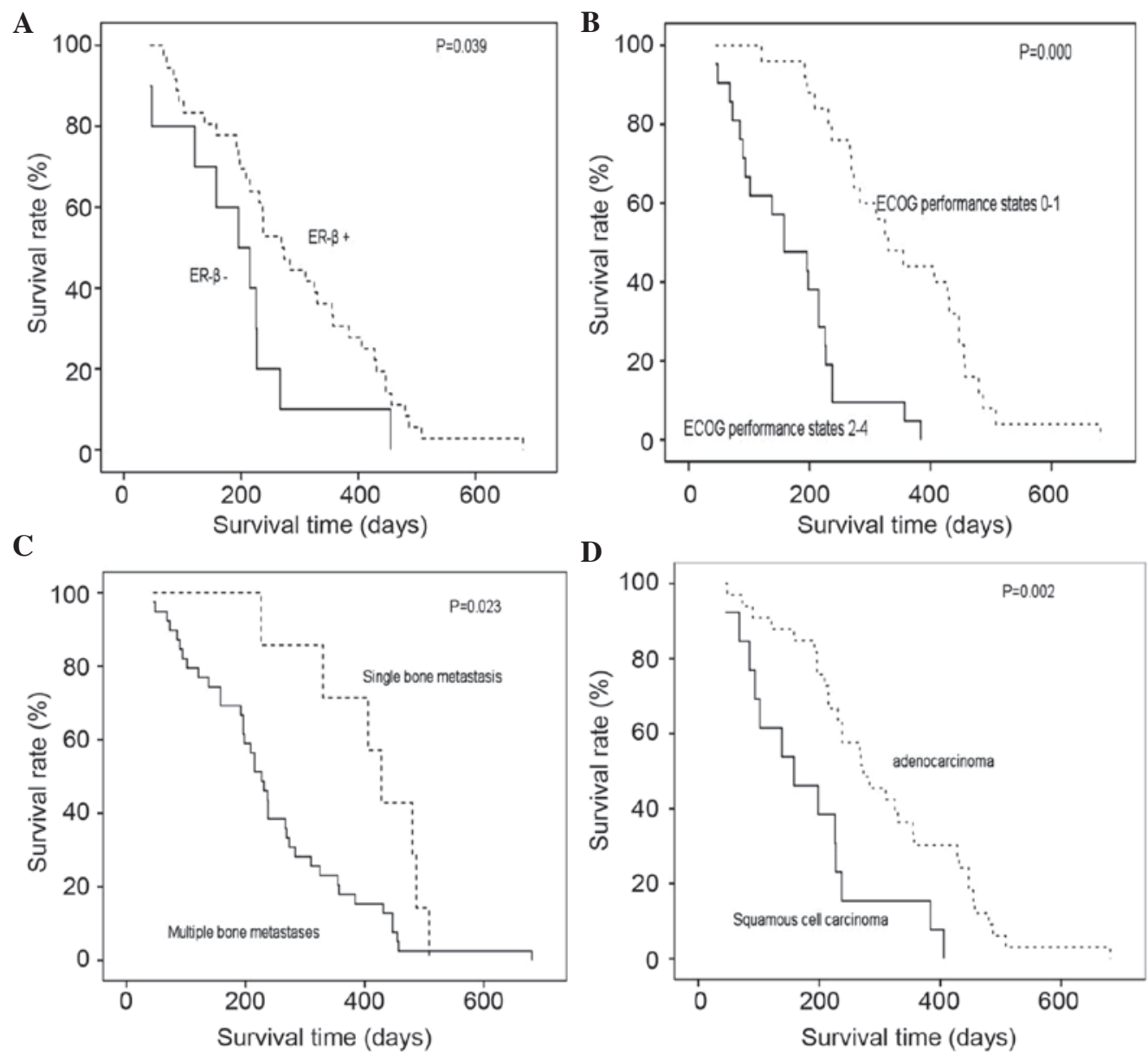

Figure 2. Kaplan-Meier survival curve of (A) estrogen receptor- $\beta$ positive and negative expression; (B) ECOG score 0-1 and 2-4; (C) single sites and more than two sites of bone metastases; and (D) adenocarcinoma and squamous cell carcinoma tumors. ECOG, Eastern Cooperative Oncology Group.

Multivariate analysis. Multivariate analysis for overall survival (OS) revealed that the pathological type $(\mathrm{P}=0.022)$, number of skeletal metastases $(\mathrm{P}=0.016)$ and $\mathrm{ER}-\beta$ expression status $(\mathrm{P}=0.035)$ were independent prognostic factors of NSCLC with bone metastases at the time of diagnosis (Table II; Fig. 2).

\section{Discussion}

Adenocarcinoma is the most frequently occurring type of NSCLC and its poor prognosis is associated with the early occurrence of distant metastases, most commonly metastasis to the bones (7). Adenocarcinoma accounted for 71.7\% (33/46) of cases in the current clinical analysis. Bone metastases may be more likely to occur in patients with lung adenocarcinoma for the following reasons: i) Adenocarcinoma is often located in the peripheral portions of the lungs, thus, it is positioned to invade the rib bones (8); and ii) adenocarcinoma easily invades the blood and lymphatic vessels due to its infiltrating and destructive growth characteristics, thus increasing the occurrence of distant metastases (9). Patients presenting with adenocarcinoma lung cancer were determined to exhibit shorter survival times compared with patients presenting with squamous cell carcinoma, as adenocarcinoma is more prone to distant metastases. However, the present study determined the opposite results, as the patients with adenocarcinoma survived significantly longer compared with the patients exhibiting 
squamous cell carcinoma ( $\mathrm{P}=0.002)$. Furthermore, multivariate analysis indicated that histological type was an independent prognostic factor of NSCLC with bone metastasis at the time of diagnosis ( $\mathrm{P}=0.037$; 95\% CI, 1.070-8.389), in agreement with results reported by Bae et al (10). Lung adenocarcinoma patients are often more sensitive to treatment with epidermal growth factor receptor-tyrosine kinase inhibitors (EGFR-TKI) following the failure of first-line chemotherapy, which may account for the differences in survival (11). Furthermore, a previous prospective study determined a higher EGFR mutation frequency rate $(51.4 \%$ overall) in Asian patients exhibiting adenocarcinoma tumors compared with squamous cell carcinoma tumors (12). In another study, although EGFR mutations were present in cases of lung squamous cell carcinoma, indicating that these patients would receive some benefit from EGFR-TKI treatment, a poorer curative effect was observed in the squamous carcinoma patients compared with the adenocarcinoma patients (13). Additional studies are required to investigate NCSLC patients with bone metastases at the time of diagnosis who are treated with EGFR-TKI, in order to ensure that such patients receive the optimal treatment regimen. Patients with single bone metastases accounted for $15.2 \%(7 / 46)$ of the present study cohort, and these patients exhibited a significantly longer survival time compared with the patients exhibiting multiple bone metastases (428 vs. 227 days; $\mathrm{P}=0.023$ ). Furthermore, Cox regression analysis identified that single bone metastases were a good independent prognostic factor $(\mathrm{P}=0.036$; $95 \% \mathrm{CI}, 1.083-9.827)$, in agreement with the results of a previous study (11).

It has previously been identified that ECOG performance status is an independent prognostic factor for lung cancer patients with bone metastases (14). In the present study, patients with an ECOG performance status of 0-1 exhibited a significantly longer OS time compared with patients scoring $2-4$, according to univariate analysis $(\mathrm{P}=0.006)$. However, the ECOG score was not an independent prognostic factor for the NSCLC patients with bone metastases at the time of diagnosis $(\mathrm{P}>0.05)$. A poor performance status was determined by the characteristics of NSCLC with bone metastasis at the time of diagnosis, including pain, spinal cord compression and fractures. However, such phenomena demonstrated marked improvement following the application of analgesic therapy, chemotherapy, local radiotherapy or surgical fixation. This was markedly different from the poor performance status caused by cachexia in patients with advanced tumors in the current study.

ER is expressed in lung cancer, however, it is not expressed in healthy lung tissues $(4,15)$. This may indicate an association between ER expression and the occurrence of lung cancer, and may provide an opportunity to develop novel antitumor agents for the treatment of NSCLC. The ER includes ER- $\alpha$, ER- $\beta$ and ER- $\gamma$. ER- $\beta$ is most commonly expressed in lung cancer and appears to be closely associated with the prognosis of lung cancer patients (16). Furthermore, estrogen promotes the proliferation of estrogen-dependent tumors, possibly via the following mechanisms (17,18): i) Estrogen action leading to tumor growth involves the activation of early response genes, such as c-myc, c-jun and c-fos, promoting DNA synthesis and cell division; ii) estrogen affects cell proliferation by adjusting cell cycle regulatory gene products and growth factors; or iii) estrogen stimulates the expression of growth factors, such as EGF and transforming growth factor- $\alpha$, and promotes cell proliferation. Aside from these mechanisms, ER- $\beta$ may play a role in the occurrence and development of lung cancer by interacting with estrogen and then via involvement in the insulin growth factor pathway (19). In the present study, 78.3\% (36/46) of patients expressed ER- $\beta$, which was a significantly higher proportion than the $45-69 \%$ reported in previous studies (20). This result may indicate that NSCLC patients with bone metastases at the time of diagnosis tend to express ER- $\beta$ more often than those without bone metastases. Additional analyses performed in the present study identified that ER- $\beta$ expression was not associated with age, gender, pathological type, number of bone metastases, the presence of other organ metastases, or serum ALK and CEA levels.

A previous study determined that the expression level of ER- $\beta$ in adenocarcinoma was significantly higher than that in squamous cell carcinomas (21), and that the majority of female patients with NSCLC exhibited adenocarcinoma, resulting in greater ER- $\beta$ expression in females compared with males. However, alternative studies identified that ER- $\beta$ also exhibited a high expression level in male patients with lung cancer (22), that ER- $\beta$ expression was higher in males compared with females (23), and that no association existed between ER- $\beta$ expression and gender in patients with NSCLC (24). Thus, the presence of an association between ER- $\beta$ expression and gender is inconclusive. In the present study, it was identified that ER- $\beta$ expression was higher in adenocarcinoma and female patients compared with squamous cell carcinoma and male patients; however, this difference was not statistically significant $(\mathrm{P}>0.05)$. This may indicate that gender and tumor type are not associated with the expression of ER- $\beta$; however, alternative possibilities cannot be dismissed due to the limited sample size.

In the present study, ER- $\beta$ expression, as well as histology and the number of bone metastases, were independent prognostic factors of NSCLC patients with bone metastases at time of diagnosis ( $\mathrm{P}=0.035$; 95\% CI, 0.167-0.939). Kaplan-Meier survival analysis demonstrated that patients with ER- $\beta$-positive expression exhibited a significantly longer OS time compared with the $E R-\beta$-negative group $(\mathrm{P}=0.039)$. It was previously reported that the expression of ER- $\beta$ may be associated with tumor differentiation, with a higher differentiation grade indicating higher expression of ER- $\beta$; thus, ER- $\beta$ expression could be used to determine prognosis (25). However, one study proposed that NSCLC patients with negative ER- $\beta$ expression were at a high risk even if they were at an early clinical stage (26). Furthermore, lack of ER- $\beta$ expression was determined to be an independent prognosis marker associated with a shorter OS time (27). Therefore, ER- $\beta$ expression levels may be used to determine the prognosis of NSCLC patients with bone metastases at the time of diagnosis.

NSCLC with bone metastasis at the time of diagnosis is an example of late-stage disease, which is associated with a poor prognosis; therefore, the present study identified a median OS time of only 237 days in this group. Platinum-based chemotherapy plus supportive care is currently the most common treatment regimen for this disease; however, its long-term curative effect is not satisfactory and easily produces drug resistance (28). Hormone therapy has been applied extensively in the treatment of breast cancer and good results have been achieved. 
Therefore, as with breast cancer, clinicians are attempting to apply hormone therapies in the treatment of NSCLC patients with ER- $\beta$-positive expression (29). Du and Jia (30) identified that hormone therapy may cause NSCLC tumor cells to arrest in the $G_{0} / G_{1}$ phase of the cell cycle by changing the endocrine environment that is required for the survival of this hormone-dependent tumor, thus improving the symptoms of such patients. The major reason why NSCLC patients with bone metastases at the time of diagnosis were insensitive to chemotherapeutic agents and tended to recur was the resistance of tumor cells to chemotherapy.

Endocrine agents target aberrant signal transduction pathways in ER-positive cancer and are involved in the mechanism of the antitumor effect. However, endocrine agents may also be considered as inhibitory agents for multi-drug resistant strains of cancer by overcoming drug resistance and increasing the sensitivity of chemotherapy. Tamoxifen is currently the most commonly used endocrine agent for the treatment of early and advanced ER-positive breast cancer, and is gradually being applied in the treatment of ER-positive NSCLC. Tamoxifen is considered to exhibit an important antitumor effect by reducing the proportion of matrix metalloproteinase-9/tissue inhibitor of metalloproteinase-1 (31). A multicenter study conducted by the International Lung Cancer Consortium (32) revealed that oral contraceptives, accepted hormone replacement therapy or a combination of estrogen and progesterone had been demonstrated to reduce the risk of lung cancer. In recent years, endocrine agents have gradually been used in the treatment of NSCLC. Endocrine agents exhibit their antitumor effect by acting in combination with traditional chemotherapeutic agents or targeted antitumor agents. For example, tamoxifen enhances erlotinib-induced cytotoxicity and increases its curative effect in patients with NSCLC (33). Therefore, estrogen agents combined with chemotherapy may improve the curative effect and tolerance of chemotherapy alone; thus, prolonging the survival time of NSCLC patients with bone metastases at the time of diagnosis.

In conclusion, in the present study, adenocarcinoma was the most common histological type of NSCLC with bone metastases at the time of diagnosis. Furthermore, the patients involved in the present study commonly exhibited metastases in other organs. ER- $\beta$-positive expression, adenocarcinoma and single bone metastasis were independent prognostic factors for the majority of patients, and may be used as prognostic indicators to assess the OS of NSCLC patients with bone metastases at the time of diagnosis. Due to the poor prognosis of such patients, additional studies regarding ER- $\beta$ expression and the efficacy of hormonal therapy are required.

\section{References}

1. Chen W, Zheng R, Zhang S, et al: Annual report on status of cancer in China, 2010. Chin J Cancer Res 26: 48-58, 2014.

2. Coleman RE: Clinical features of metastatic bone disease and risk of skeletal morbidity. Clin Cancer Res 12: 6243-6249, 2006.

3. Tsuya A, Kurata T, Tamura K, et al: Skeletal metastases in non-small cell lung cancer: a retrospective study. Lung Cancer 57: 229S-232S, 2007.

4. Beattie CW, Hansen NW and Thomas PA: Steroid receptors in human lung cancer. Cancer Res 45: 4206-4214, 1985.

5. Santarpia L, Koch CA and Sarlis NJ: Hypercalcemia in cancer patients: pathobiology and management. Horm Metab Res 42: 153-164, 2010.
6. Oken MM, Creech RH, Tormey DC, et al. Toxicity and response criteria of the Eastern Cooperative Oncology Group. Am J Clin Oncol 5: 649-55, 1982

7. Krawczyk P, Nicoś M, Ramlau R, et al: The incidence of EGFR-activating mutations in bone metastases of lung adenocarcinoma. Pathol Oncol Res 20: 107-112, 2014.

8. Lee Y, Chung JH, Kim SE, et al: Adenosquamous carcinoma of the lung: CT, FDG PET, and clinicopathologic findings. Clin Nucl Med 39: 107-112, 2014.

9. Yuan L, Zhang J, Tan H, et al: An analysis of 1657 inpatients with lung cancer from 2001 to 2008 . Ning xia yi ke da xue xue bao 32: 374-377, 2010.

10. Bae HM, Lee SH, Kim TM, et al: Prognostic factors for non-small cell lung cancer with bone metastasis at the time of diagnosis. Lung Cancer 77: 572-577, 2012.

11. Zhang Y, Jin B, Shao M, et al. Monitoring of carcinoembryonic antigen levels is predictive of EGFR mutations and efficacy of EGFR-TKI in patients with lung adenocarcinoma. Tumour Biol 35: 4921-8, 2014.

12. Shi Y, Au JS, Thongprasert S, et al: A prospective, molecular epidemiology study of EGFR mutations in Asian patients with advanced non-small-cell lung cancer of adenocarcinoma histology (PIONEER). J Thorac Oncol 9: 154-162, 2014.

13. Duan JC, An TT, Wu MN, et al: Correlation between the efficacy of epidermal growth factor receptor tyrosine kinase inhibitors and EGFR mutations in advanced squamous cell lung cancer. Zhonghua Jie He He Hu Xi Za Zhi 35: 323-328, 2012 (In Chinese)

14. Ohashi R, Takahashi K, Miura K, et al: Prognostic factors in patients with inoperable non-small cell lung caner - an analysis of long-term survival patients. Gan To Kagaku Ryoho 33: 1595-1602, 2006.

15. Ivanova M, Abner S, Pierce W Jr and Klinge C: Ligand-dependent differences in estrogen receptor beta-interacting proteins identified in lung adenocarcinoma cells corresponds to estrogenic responses. Proteome Sci 9: 60, 2011.

16. Monica V, Longo M, Felice B, et al: Role of hormone receptor expression in patients with advanced-stage lung cancer treated with chemotherapy. Clin Lung Cancer 13: 416-423, 2012.

17. Pietras RJ, Márquez DC, Chen HW, et al: Estrogen and growth factor receptor interactions in human breast and non-small cell lung cancer cells. Steroids 70: 372-381, 2005.

18. Stabile LP, Lyker JS, Gubish CT, et al: Combined targeting of the estrogen receptor and the epidermal growth factor receptor in non-small cell lung cancer shows enhanced antiproliferative effects. Cancer Res 65: 1459-1470, 2005.

19. Tang H, Liao Y, Chen G, et al: Estrogen upregulates the IGF-1 signaling pathway in lung cancer through estrogen receptor- $\beta$. Med Oncol 29: 2640-2648, 2012.

20. Siegfried JM, Hershberger PA and Stabile LP: Estrogen receptor signaling in lung cancer. Semin Oncol 36: 524-531, 2009.

21. Rodriguez-Lara V,Peña-Mirabal E, Baez-Saldaña R, et al: Estrogen receptor beta and CXCR4/CXCL12 expression: differences by sex and hormonal status in lung adenocarcinoma. Arch Med Res 45:158-169, 2014.

22. Siegfried JM: Smoking out reproductive hormone actions in lung cancer. Mol Cancer Res 12: 24-31, 2014.

23. Schwartz AG, Prysak GM, Murphy V, et al: Nuclear estrogen receptor $\beta$ in lung cancer: expression and survival differences by sex. Clin Cancer Res 11: 7280-7287, 2005.

24. Navaratnam S, Skliris G, Qing G, et al: Differential role of estrogen receptor beta in early versus metastatic non-small cell lung cancer. Horm Cancer 3: 93-100, 2012.

25. Lixian Z: Serum estrogen levels of lung cancer patients and estrogen receptor expression in lung cancer tissue and its clinical pathological significance (unpublished $\mathrm{PhD}$ thesis). Fujian Medical University, 2013.

26. Kawai H, Ishii A, Washiya K, et al: Estrogen receptor alpha and beta are prognostic factors in non-small cell lung cancer. Clin Cancer Res 11: 5084-5089, 2005.

27. Mauro LV, Dalurzo M, Carlini MJ, et al: Estrogen receptor $\beta$ and epidermal growth factor receptor as early-stage prognostic biomarkers of non-small cell lung cancer. Oncol Rep 24: 1331-1338, 2010.

28. Rossi A, Chiodini P, Sun JM, et al: Six versus fewer planned cycles of first-line platinum-based chemotherapy for non-small-cell lung cancer: a systematic review and meta-analysis of individual patient data. Lancet Oncol 15: 1254-1262, 2014.

29. Gallo D, De Stefano I, Grazia Prisco M, et al: Estrogen receptor beta in cancer: an attractive target for therapy. Curr Pharm Des 18: 2734-2757, 2012. 
30. Du KQ and Jia CX: The expression of estrogen receptor and endocrine therapy of non-small cell lung cancer with the expression of estrogen receptor and endocrine therapy. Acta Acad Med Xuzhou 30: 278-280, 2010.

31. Wang XY, Wang Y and Liu HC: Tamoxifen lowers the MMP-9/TIMP-1 ratio and inhibits the invasion capacity of ER-positive non-small cell lung cancer cells. Biomed Pharmacother 65: 525-528, 2011.

32. Pesatori AC, Carugno M, Consonni D, et al: Hormone use and risk for lung cancer: a pooled analysis from the International Lung Cancer Consortium (ILCCO). Br J Cancer 109: 1954-1964, 2013.
33. Ko JC, Chiu HC, Syu JJ, et al: Tamoxifen enhances erlotinib-induced cytotoxicity through down-regulating AKT-mediated thymidine phosphorylase expression in human non-small-cell lung cancer cells. Biochem Pharmacol 88: $119-127,2014$. 